\title{
ON THE FIXED POINT INDEX OF ITERATES OF PLANAR HOMEOMORPHISMS
}

\author{
MORTON BROWN
}

(Communicated by R. Daniel Mauldin)

\begin{abstract}
If $f$ is an orientation preserving homeomorphism of the plane with an isolated fixed point at the origin 0 and $\operatorname{index}(f, 0)=p$, then $\operatorname{index}\left(f^{n}, 0\right)$ is always well defined provided that $p \neq 1$. In this case, for each $n \neq 0$, index $\left(f^{n}, 0\right)=\operatorname{index}(f, 0)=p$. If $\operatorname{index}(f, 0)=1$, then there is an integer $p$ (possibly $p=1$ ) such that for those values of $n$ for which $\operatorname{index}\left(f^{n}, 0\right)$ is defined (i.e 0 is an isolated fixed point of $\left.f^{n}\right), \operatorname{index}\left(f^{n}, 0\right)=1$ or index $\left(f^{n}, 0\right)=p$.
\end{abstract}

\section{INTRODUCTION}

If 0 is an isolated fixed point for the continuous map $f: U \rightarrow R^{m}$ where $U$ is an open subset of $R^{m}$, then the index of $f$ at $0, \operatorname{index}(f, 0)$, is the local degree of the mapping Id $-f$ restricted to an appropriately small open set about 0 . If 0 is an isolated fixed point of $f^{n}$, then $\operatorname{ind}\left(f^{n}, 0\right)$ is defined for all $n>0$, where $f^{n}$ means $f$ composed with itself $n$ times restricted to a small neighborhood of 0 . Shub and Sullivan [SS] observed that if $f: U \rightarrow R^{m}$ is $C^{1}$ and 0 is an isolated point of $f^{n}$ for all $n$, then $\operatorname{index}\left(f^{n}, 0\right)$ is bounded as a function of $n$. They also provided a simple counterexample if the condition that $f$ be $C^{1}$ is weakened to: $f$ is continuous. The purpose of this paper is to show that for homeomorphisms a similar (stronger) result obtains in the case $m=2$.

Our principal results are as follows: If $f$ is an orientation preserving homeomorphism of the plane with an isolated fixed point at the origin 0 and index $(f, 0)=p$, then $\operatorname{index}\left(f^{n}, 0\right)$ is always well defined provided that $p \neq 1$. In this case, for each $n \neq 0, \operatorname{index}\left(f^{n}, 0\right)=\operatorname{index}(f, 0)=p$. If $\operatorname{index}(f, 0)=$ 1 , then there is an integer $p$ (possibly $p=1$ ) such that for those values of $n$ for which $\operatorname{index}\left(f^{n}, 0\right)$ is defined (i.e 0 is an isolated fixed point of $f^{n}$ ), $\operatorname{index}\left(f^{n}, 0\right)=1$ or $\operatorname{index}\left(f^{n}, 0\right)=p$. In another paper we shall show that if $f$ is orientation reversing and has an isolated fixed point at the origin, then index $(f, 0)$ is one of the three values $0, \pm 1$.

Received by the editors April 4, 1989 and, in revised form, May 16, 1989.

1980 Mathematics Subject Classification (1985 Revision). Primary 58F20, 54H25. 
In $\S 2$, we develop the main results in their conceptually simplest form. These are theorems about orientation preserving homeomorphisms of the plane with exactly one fixed point. In $\S 3$, we treat the generic (localized) situation of an isolated fixed point.

2.

Definition 1. The flows whose phase portraits are described in Figure 1 are the "canonical flows" of index $p>1$ as in Figure $1 \mathrm{a}, p<1$ as in Figure $1 \mathrm{~b}$ and $p=1$ as in Figure 1c. The canonical flow of index $p$ has $2(1-p)$ hyperbolic
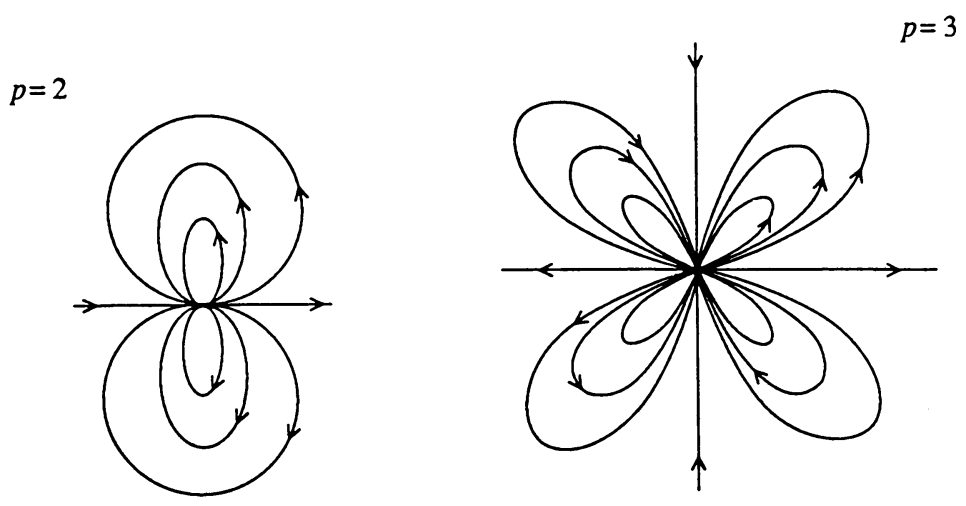

Figure 1a.
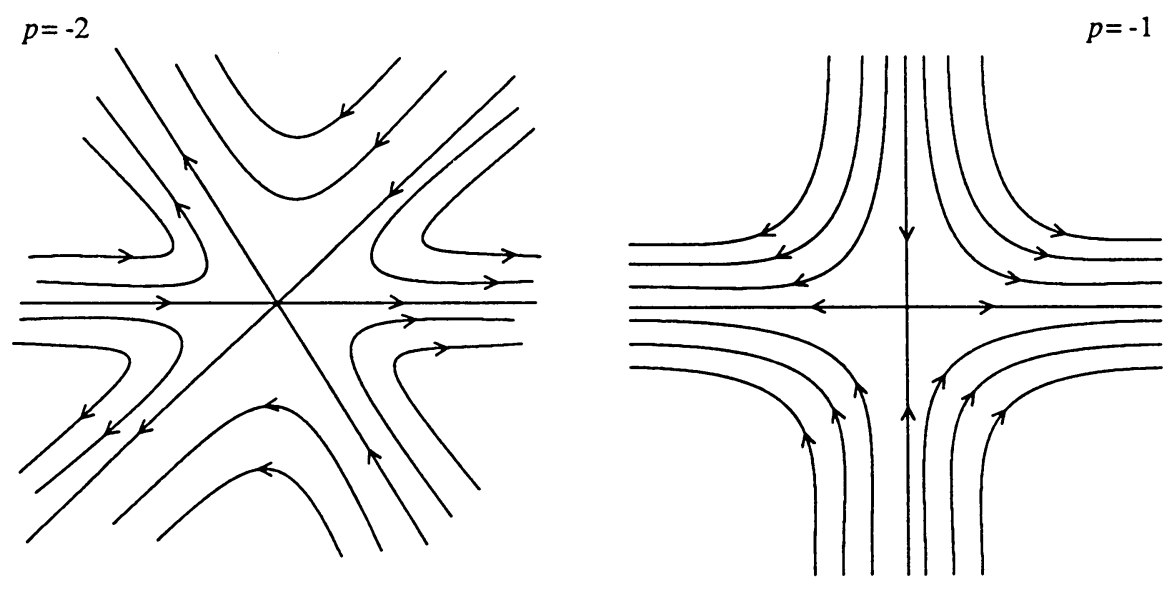

Figure $1 \mathrm{~b}$. 


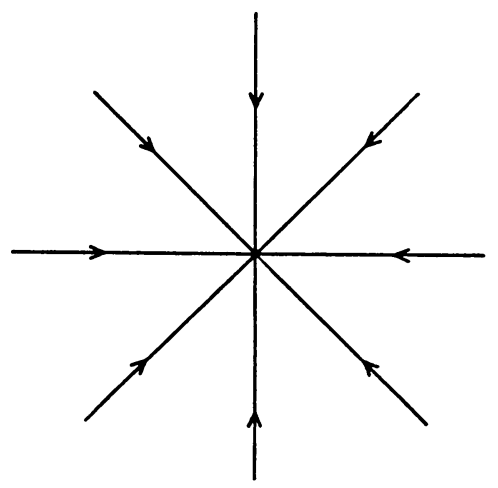

FigURE 1c.

regions if $p<1$, and $2(p-1)$ elliptic regions if $p>1$. For each $p$ we will denote by $h_{p}$ the time one homeomorphism of the canonical flow of index $p$. Any conjugate of the homeomorphism $h_{p}$ has the same phase portrait, and we will refer to any one of these as a canonical homeomorphism of index $p$. The restriction of the homeomorphism $h_{p}$ to the unit circle $J$ has image $h(J)=K$ as described in Figure 2 where for each $i$, the inverse of the $\operatorname{arc} a_{i}$ is the $\operatorname{arc} b_{i}$. If $p<1$ then there will be $(1-p)$ arcs $a_{i}$. If $p>1$ there will be $(p-1)$ arcs $a_{i}$. Obviously, since $h_{p}$ is the time one homeomorphism of the canonical flow of index $p$ then the index of $h_{p}^{n}$ on $J$ (that is, the index of the $n$th iterate of $h_{p}$ restricted to $J$ ) $=$ (the index of the time $n$ homeomorphism of the canonical flow of index $p$ ) $=p$.
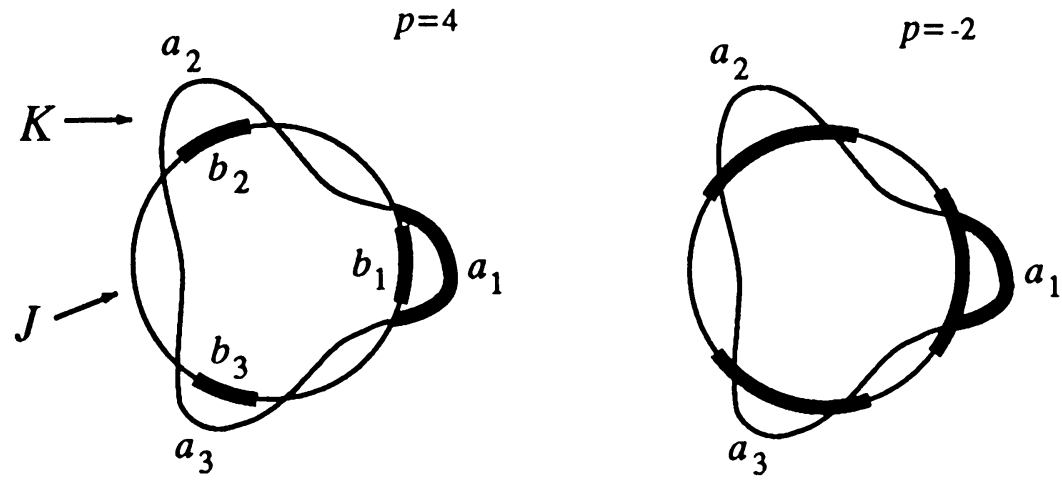

Figure 2. 
Theorem 1 [S]. Let $f$ be an orientation preserving homeomorphism of the plane such that the origin 0 is the only fixed point of $f$, and the index of $f$ at 0 is $p$. Then there is an isotopy of $h$ to a canonical homeomorphism $h_{p}$. Throughout the isotopy the intermediate homeomorphisms have 0 as their only fixed point.

Definition 2. A homeomorphism $f$ of the plane is "free" provided that whenever $D$ is a topological disk in the plane such that $f(D) \cap D=\Phi$, then for all $i \neq j, f^{i}(D) \cap f^{j}(D)=\varnothing$. Clearly, the only periodic points of a free homeomorphism are fixed points.

Definition 3. If $f$ is a homeomorphism of an open subset $U$ of the plane into the plane and $J$ is a simple closed curve in $U$ such that $f$ has no fixed points on $J$ then the index of $f$ on $J$, index $(f, J)$ is defined as the degree of the map $(f-\mathrm{id}) /\|f-\mathrm{id}\|$ from $J$ to the unit circle. Recall that index $(f, J)$ is invariant under a homotopy $h_{t}\left(J_{t}\right)$ as long as (1) $h_{t}$ is fixed point free on $J_{t}$ throughout, (2) index $(f, J)=\operatorname{index}\left(f^{-1}, f(J)\right)$, and (3) if 0 is an isolated fixed point of $f$ then index $(f, 0)=\operatorname{index}(f, J)$ for any simple closed curve $J$ which surrounds 0 and no other fixed point of $f[\mathrm{BN}]$.

Theorem 2 (Brown, $\left.\left[\mathrm{B}_{2}\right]\right)$. Let $f$ be an orientation preserving homeomorphism of the plane. If $f$ is not free, then there is a simple closed cuve $J$ in the plane such that $\operatorname{index}(f, J)=1$.

Corollary. Let $f$ be an orientation preserving homeomorphism of the plane with a single fixed point 0 . Suppose the index of $f$ at 0 is $p \neq 1$. Then $f$ is free, and hence has no periodic points other than the fixed point 0 .

Proof. If $J$ is a simple closed curve such that the origin is not a point of $J$, then either $\operatorname{index}(f, J)=p$ or $\operatorname{index}(f, J)=0$, depending upon whether or not $J$ contains the origin in its interior. In either case index $(f, J) \neq 1$.

Theorem 3. Let $f$ be an orientation preserving homeomorphism of the plane with the origin 0 as its unique fixed point. Suppose $\operatorname{index}(f, 0)=p$, and $p \neq 1$. Then for all $n \neq 0$, index $\left(f^{n}, 0\right)=\operatorname{index}(f, 0)$.

Proof. According to Theorem 1, $f$ is isotopic to a canonical homeomorphism $h_{p}$ through an isotopy $g_{t}\left(0 \leq t \leq 1, g_{1}=h_{p}, g_{0}=f\right)$ where $g_{t}$ has 0 as a unique fixed point. Thus the index of $g_{t}$ is $p$ for all $t$, so that each $g_{t}$ is free, and thus $g_{t}$ has no periodic points other than the origin. This implies that $g_{t}^{n}$ has only the origin as a fixed point. Thus $g_{t}^{n}$ is an isotopy from $f^{n}$ to $h_{p}^{n}$ where $g_{t}^{n}$ has only 0 as a fixed point. Thus index $\left(f^{n}, 0\right)=\operatorname{index}\left(h_{p}^{n}, 0\right)=p$, the last equality coming from the remarks following Definition 1.

Remark. What goes wrong when $p=1$ ? Let $r$ denote the rotation of the plane by $\pi$, let $h$ be the standard "Anosov" homeomorphism $\left(h=h_{-1}\right): h(x, y)=$ $(2 x, y / 2)$, and let $f=h r$. By direct calculation index $(f, 0)=1$. On the other hand, $f^{2}=h^{2}$, so index $\left(f^{2}, 0\right)=-1$. Note that the canonical homeomorphism $h_{1}$ maps the unit circle strictly into its interior, so by Theorem $1, f$ 
is isotopic to this map via homeomorphisms whose only fixed point is 0 . This can be accomplished easily with $f$ by simply isotoping $f$, say by $f_{t}$ along horizontal lines until the $f$-image of the unit circle lies inside its interior. It is easily seen that $f_{t}$ has only the origin as a fixed point. Since $f^{2}$ and $h^{2}$ have different indexes, we must conclude that some points of period 2 are introduced during the isotopy. It follows from the proof of Theorem 3 that any isotopy of $f$ to $h_{1}$ must introduce fixed points or points of period two during the isotopy.

Theorem 4. Suppose $f$ is a homeomorphism of the plane with 0 as its unique fixed point. Suppose that index $(f, 0)=1$. Then either $\operatorname{index}\left(f^{n}, 0\right)=1$ for every $n$ for which index $\left(f^{n}, 0\right)$ is defined, or there is an integer $p$ such that for each $n$, either index $\left(f^{n}, 0\right)$ is not defined (i.e 0 is not an isolated fixed point of $\left.f^{n}\right)$, or $\operatorname{index}\left(f^{n}, 0\right)=1$ or $\operatorname{index}\left(f^{n}, 0\right)=p$.

Proof. Obviously, if index $\left(f^{n}, 0\right)=p$, and index $\left(f^{m}, 0\right)=q$, and neither $p$ nor $q$ is equal to 1 , then by Theorem 3 ,

$$
p=\operatorname{index}\left(f^{n}, 0\right)=\operatorname{ind}\left(f^{n m}, 0\right)=\operatorname{index}\left(f^{m}, 0\right)=q .
$$

Remark. All situations described in Theorem 4 can occur. If $f$ is the composition of the canonical index $p$ homeomorphism $(p>2)$ with a rotation by $2 \pi /(p-1)$ then $f$ has index 1 for $1 \leq n \leq p-1$, and $\operatorname{index}\left(f^{n}, 0\right)=$ index $\left(h_{p}\right)^{n}=\operatorname{index}\left(h_{p}, 0\right)=p$. A similar construction obtains for $p<0$. (I do not know of examples where $p=0$ or 2.) On the other hand, if $f$ is a rotation by an irrational multiple of $2 \pi$ then index $\left(f^{n}, 0\right)=1$ for all $n$. Finally if $f$ is a "rational" rotation, then for certain values of $f$ and $n, 0$ is not an isolated fixed point of $f^{n}$.

\section{3.}

In this section we study orientation preserving homeomorphisms of the plane in which the origin is an isolated fixed point. We do not assume that the origin is isolated as a periodic point. Nevertheless, we obtain results similar to those of $\S 1$. It will be necessary at times, in order to get the slightly stronger results that are needed, to refer to the methods of $[S],\left[B_{1}\right]$, and $\left[B_{2}\right]$. Theorem 5, for example, is a slightly refined version of Theorem 5.7 of $\left[\mathrm{B}_{2}\right]$. The reader who wishes to verify this should retrace the argument in $\left[\mathrm{B}_{1}\right]$ or $\left[\mathrm{B}_{2}\right]$.

Theorem 5. Let $f$ be a homeomorphism of an open set $U$ of the plane into the plane. Suppose $x$ is a point of $U$ such that (1) $f(x) \neq x$, (2) for some $n>1, f^{i}(x)$ is in $U$ for $1 \leq i \leq n$, and (3) $f^{n}(x)=x$. Suppose there exists a connected neighborhood $W$ of $\{x\} \cup\{f(x)\}$ such that $f^{i}(W)$ is a subset of $U$ for $1 \leq i \leq n$, and $W$ contains no fixed points of $f$. Then there is a simple closed curve $J$ in $U$ such that $\operatorname{index}(f, J)=1$.

Theorem 6. Let $f$ be an orientation preserving homeomorphism of the plane such that 0 is an isolated fixed point of $f$ and $\operatorname{index}(f, 0)=p \neq 1$. Then for each $n>0,0$ is an isolated fixed point of $f^{n}$. 
Proof. Let $n>0$ be given, and let $U$ be a neighborhood of 0 containing no fixed points of $f$ other than 0 . Then the index of $f$ on any simple closed curve in $U$ is either $p$ or zero. Let $V$ be a connected neighborhood of 0 such that $f^{i}(V)$ is a subset of $U$ for each $i, 1 \leq i \leq n+1$. Let $x$ be a point of $V$ other than 0 . If $f^{i}(x)=x$ for $1<i \leq n$, then by Theorem 5 , with $W=V \cup f(V), U$ contains a simple closed curve on which the index of $f$ is 1 . This contradicts the assumption that $p \neq 1$.

Remark. One must choose $V$ after $n$ is given, since 0 might not be isolated as a periodic point. Smale's horseshoe provides such an example.

Theorem 7 [S]. Let $f$ be a homeomorphism of the plane such that 0 is an isolated fixed point of $f$ and index $(f, 0)=p$. Let $U$ be a neighborhood of 0 . Then there is a neighborhood $V$ of the origin and an isotopy $h_{t}$ connecting $f$ to a homeomorphism $g$ such that (1) $g \mid v$ is canonical of index $p$, (2) for all $t, 0 \leq t \leq 1, h_{t}\left|\left(R^{2}-U\right)=f\right|\left(R^{2}-U\right)$, and (3) $h_{t} \mid U$ has 0 as a unique fixed point.

Remark. This is a modified version of the main theorem of [S]. (In paragraph 4, p. 239 apply the Alexander isotopy on one side only.) As a consequence of Theorems 5-7 and the methods of $\S 1$ we have Theorem 8 .

Theorem 8. The conclusions of Theorem 4 hold if we assume only that $f$ has 0 as an isolated fixed point instead of a unique fixed point.

\section{REFERENCES}

[B 1 ] M. Brown, A new proof of Brouwer's Lemma on translation arcs, Houston J. Math. 10 (1984), 35-41.

$\left[\mathrm{B}_{2}\right]$ _ Homeomorphisms of two-dimensional manifolds, Houston J. Math. 11 (1985), 455-469.

[BN] M. Brown and W. Neumann, Proof of the Birkhoff-Poincare fixed point theorem, Michigan Math. J. 24 (1977), 21-31.

[S] Bruno V. Schmitt, L'espace des homeomorphismes du plan qui admettant un seul point fixe d'indice donne est connexe par arcs, Topology 18 (1979), 235-240.

[SS] M. Shub and D. Sullivan, A remark on the Lefschetz fixed point formula for differentiable maps, Topology 13 (1974), 189-191.

Department of Mathematics, University of Michigan, Ann Arbor, Michigan 48109 